\title{
Gastroenterological disorders increase the prevalence of overactive bladder in females at various ages
}

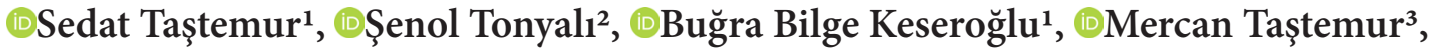 \\ ๑D Mustafa Karaaslan ${ }^{4}$, @Bayram Yeșil ${ }^{5}$, @Mahmut Yüksel ${ }^{5}$ \\ ${ }^{1}$ University of Health Sciences, Ankara City Hospital, Department of Urology, Ankara, Turkey \\ ${ }^{2}$ İstanbul University İstanbul School of Medicine, Department of Urology, İstanbul, Turkey \\ ${ }^{3}$ University of Health Sciences, Ankara City Hospital, Department of Geriatrics, Ankara, Turkey \\ ${ }^{4}$ Bingöl State Hospital, Department of Urology, Bingol, Turkey \\ ${ }^{5}$ University of Health Sciences, Ankara City Hospital, Department of Gastroenterology, Ankara, Turkey
}

Cite this article as: Taştemur S, Tonyalı Ş, Keseroğlu BB, et al. Gastroenterological disorders increase the prevalence of overactive bladder in females at various ages. J Health Sci Med 2022; 5(1): 84-87.

\begin{abstract}
Aim: In this study we aimed to determine the coexistence of overactive bladder in patients with gastroenterological disorders including hepatitis, cirrhosis and inflammatory bowel disease (IBD).

Material and Method: We prospectively collected the data of patients who admitted to the outpatient clinic of department of gastroenterology at Ankara Yüksek İhtisas Training and Research Hospital between May 2017 and February 2019. All patients with chronic gastroenterological disorders such as hepatitis and irritable bowel syndrome willing to participate after the verbal consent were included in the study

Results: A total of 289 female patients were included the study. The mean age of the patients was $49.9 \pm 13$ years. The mean BMI value was $27.9 \pm 4.5 \mathrm{~kg} / \mathrm{m}^{2}$. Among 289 patients, $135(46.7 \%)$ had Hepatitis B, $53(18.3 \%)$ had ulcerative colitis, 35 (12.1\%) had Crohn's disease, 22 (7.6\%) had autoimmune hepatitis, 19 (6.6\%) had primer biliary cirrhosis, 13 (4.5\%) had Hepatitis C and $12(4.2 \%)$ had celiac disease. The mean age of patients was similar between patients having OAB-v8 higher and lower than $8(\mathrm{p}=0.46)$. However, patients having OAB score $>8$ had higher BMI compared to patients who had OAB score $<8,29.1 \pm 5$ vs. $27.2 \pm 4.1 \mathrm{~kg} / \mathrm{m}^{2}, \mathrm{p}=0.001$. In multivariate regression analysis, $\mathrm{BMI}$ was the sole indicator of $\mathrm{OAB}(\mathrm{p}=0.001)$ whereas age $(\mathrm{p}=0.46)$, menopause status $(\mathrm{p}=0.33)$, smoking status $(\mathrm{p}=0.97)$ were not.

Conclusion: The incidence of $\mathrm{OAB}$ in our patient cohort was higher than the reported incidence by that evaluating the patients with gastrointestinal disorders in terms of overactive bladder on routine follow-up might be suggested.
\end{abstract}

Keywords: Gastroenterological disorders, OAB-v8, overactive bladder

\section{INTRODUCTION}

Overactive bladder $(\mathrm{OAB})$ is defined as urinary urgency with or without incontinence, usually accompanied with frequency and nocturia. $\mathrm{OAB}$ affects approximately $9-43 \%$ of the women population (1). However, a few people with $\mathrm{OAB}$ aspire for medical care for this disorder. $\mathrm{OAB}$ might lead to several health problems such as depression, sleep disorder and poor quality of life (2).

Ulcerative colitis and Chron's disease, known as IBD, constitute a major health burden and have detrimental effects on quality of life of affected patients. Since it is a chronic disease with unknown/ multifactorial etiology, several attempts and investigations have been held to control these inflammatory processes $(3,4)$. Currently

accepted treatment choices include antibiotics, probiotics, folic acid antagonists, aminosalicylates, corticosteroids, thiopurines, methotrexate and anti-TNF agents $(3,5)$.

It has been estimated that approximately $2.5-3$ million people are affected by IBD in Europe and there is still a tendency of increase in its incidence not only in Eastern Europe but also in Asia. The cumulative surgery rate was reported to vary between $37 \%$ and $61 \% 10$ year after diagnosis and surgery rate was found to be declined in the last two decades (5). Inflammatory bowel disease is not only a bowel limited disease but also has several extra-intestinal signs and symptoms. Extraintestinal manifestations include Joint manifestations (arthropathies), cutaneous 
manifestations (erythema nodosum, pyoderma gangrenosum), ocular manifestations which are found be frequent as high as $40 \%(5)$.

Furthermore, non-alcoholic fatty liver disease (NAFLD) was found to be associated with overactive bladder in women. The mechanism in this association was mainly based on NAFLD as being a component of metabolic syndrome. Thus, NAFLD might lead to atherosclerosis. However, mediators called hepatokines might also cause an inflammatory environment $(6,7)$. There is currently not much study examining the relationship between hepatitis and overactive bladder.

To the best of our knowledge there is no study in the literature assesing the patients having IBD or liver diseases and bladder-related comorbidities in Turkish population. Thus, in this study we aimed the determine the coexistence of bladder disorders in patients that have gastroenterological disorders including hepatitis, cirrhosis and IBD.

\section{MATERIAL AND METHOD}

The study was carried out with the permission of Health Sciences University Ankara Yüksek İhtisas Training and Research Hospital Ethics Committee (Date: 28.12.2018, Decision No: 65). All procedures were carried out in accordance with the ethical rules and the principles of the Declaration of Helsinki.

We prospectively collected the data of patients who admitted to the outpatient clinic of department of gastroenterology at Ankara Yüksek İhtisas Training and Research Hospital between May 2017 and February 2019. All patients with chronic gastroenterological disorders such as hepatitis and irritable bowel syndrome willing to participate the study after the verbal consent were included the study. The inclusion criteria were as follow:

1. Both male and female patients older than age of 18

2. Having chronic gastroenterological disorders

3. Willing to participate the study and fulfill the questionnaire

4. Having regular follow-up.

The exclusion criteria included:

1. Being younger than 18 years of old.

2. Having a history of cancer

3. Receiving any chemo-radiotherapy for any reasons.

4. Being on medications like opioids or analgesics.

5. Having a pelvic organ prolapse

6. Having had surgery for cystocele or stress urinary incontinence

7. Having psychiatric disorders impairing or aggregating pain perception.
All patients were requested to fulfill the Overactive bladder V8 questionnaire which is a validated questionnaire in Turkish (8). The recorded parameters also included patient demographics, medical history and medications, complete blood count, urinalysis, serum urea and creatinine. The patients were divided into three groups according to symptom severity as in previous studies: Group 1- having OAB v8 score $=0-7$, Group 2having $\mathrm{OAB}$ v8 score=8-16, Group 3- having OAB-v8 $>16$ (9). Patients having $\mathrm{OAB}$ v8 score $>7$ assumed to have OAB.

\section{Statistical Analysis}

Statistical analysis was performed using IBM SPSS Statistics for Windows v.21.0 (IBM Corp., Armonk, NY). Quantitative values are shown as mean \pm SD (range) and qualitative values are shown as number and percentage. One-way ANOVA and the chi-squared test were used to compare dichotomous variables between groups. Student's t-test and the Mann-Whitney U-test were used to compare groups with normally and non-normally distributed continuous data, respectively. The level of statistical significance was set at $\mathrm{p}<.05$.

\section{RESULTS}

A total of 289 female patients were included the study. The mean age of the patients was $49.9 \pm 13$ years. The mean body mass index (BMI) was $27.9 \pm 4.5 \mathrm{~kg} / \mathrm{m}^{2}$. Among 289 patients, 135 (46.7\%) had Hepatitis B, 53 (18.3\%) had ulcerative colitis, 35 (12.1\%) had Crohn's disease, 22 (7.6\%) had autoimmune hepatitis, 19 (6.6\%) had primer biliary cirrhosis, 13 (4.5\%) had Hepatitis $\mathrm{C}$ and 12 (4.2\%) had celiac disease. 115 (39.8\%) of the patients were in menopause. $31(10.7 \%)$ were active smokers. Six patients $(2.1 \%)$ had psychiatric disease including depression $(\mathrm{n}=3)$, anxiety $(\mathrm{n}=2)$ and panic attack $(n=1) .78$ patients $(22.8 \%)$ had a OAB-v8 score higher than 8 (Table 1).

There was no statistically difference between patients having different gastroenterological pathologies in terms of OAB ( $\mathrm{p}=0.34)$. The mean age of patients was similar between patients having OAB-v8 higher and lower than $8(\mathrm{p}=0.46)$. However, patients having $\mathrm{OAB}$ score $>8$ had higher BMI compared to patients who had $\mathrm{OAB}$ score $<8,29.1 \pm 5$ vs. $27.2 \pm 4.1 \mathrm{~kg} / \mathrm{m}^{2}, \mathrm{p}=0.001$. There was no difference between patients who had menopause and who had not in terms of OAB score, $\mathrm{p}=0.37$. OAB frequency was similar between patients who were active smoker and who were not active smokers $(\mathrm{p}=0.11)$. In multivariate regression analysis, $\mathrm{BMI}$ was the sole indicator of $\mathrm{OAB}(\mathrm{p}=0.001)$ whereas age $(\mathrm{p}=0.46)$, menopause status $(\mathrm{p}=0.33)$, smoking status $(\mathrm{p}=0.97)$ were not (Table 1$)$. 


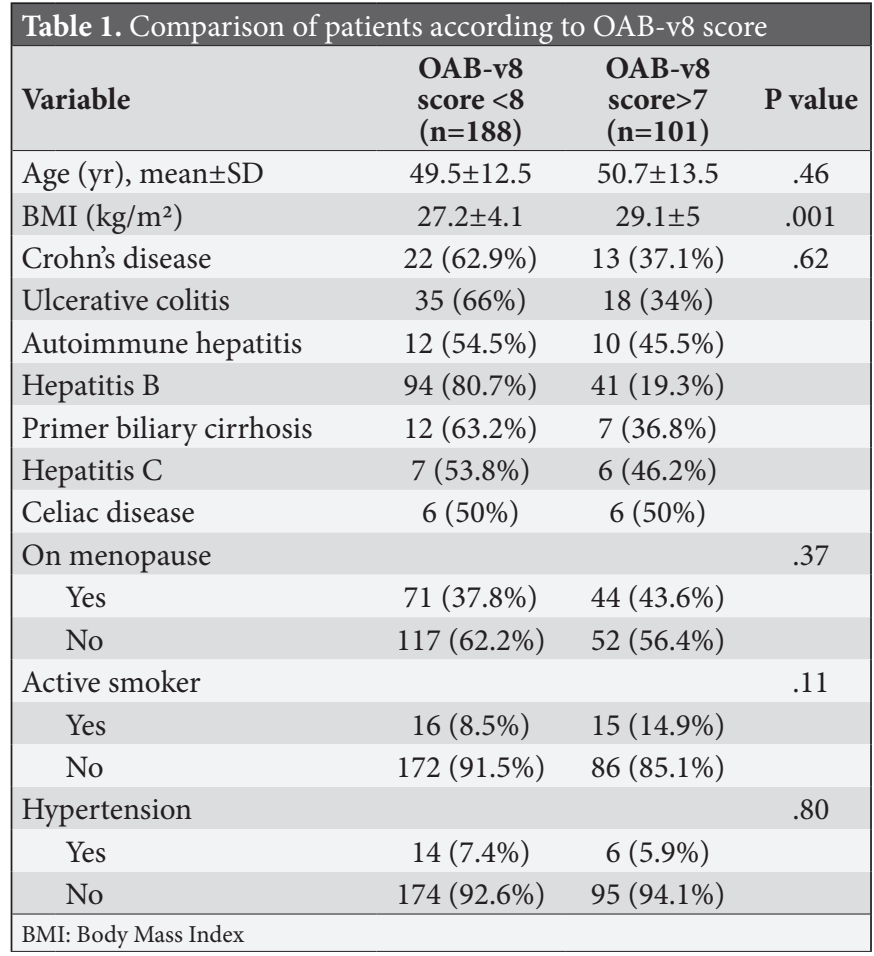

\section{DISCUSSION}

$\mathrm{OAB}$ is defined as urgency with or without incontinence, often accompanied with frequency or nocturia. $\mathrm{OAB}$ may be idiopathic or related to neurologic disorders. The reported prevalence of $\mathrm{OAB}$ varies between 5.9 and $15.6 \%$ and strongly correlated with aging. $\mathrm{OAB}$ severity tends to increase in post-menopausal women which might be associated with decreased levels of estrogen (10). In our patient cohort, the prevalence of OAB was $22.8 \%$ which might indicate the high prevalence of $\mathrm{OAB}$ related to gastrointestinal disorders.

Etiology of $O A B$ is considered multifactorial and ischemia related bladder dysfunction is one of the suggested mechanisms. It has been reported that atherosclerosis causes ischemia in bladder. Smoking is one of the leading factor of atherosclerosis. In previous studies, $\mathrm{OAB}$ symptoms have been shown to strongly correlated with aging and smoking (11).

Increased BMI was also reported to be associated with OAB symptoms(12). In this study, the mean age of the patients and smoking status of the patient who had $O A B$ symptoms and who had not, were similar. This might be occurred due to the confounder effect of gastrointestinal disorders.

Cross organ sensitization has been widely studied and suggested in various conditions including trochal and abdominal organs. The organs reported to involve in cross organ sensitization phenomena including pelvic and lower abdominal organs: colon, rectum, urinary bladder, urethra, uterus and the prostate. It has been also suggested that urinary bladder is more vulnerable to cross-sensitization than other pelvic organs (13). In an former study in 1980's Whoewel et al. (14) reported that patient with irritable bowel syndrome may represent with symptoms related to urinary bladder such as nocturia, frequency, urgency and sense of incomplete bladder emptying. Gastrointestinal disorders generally classified under two groups as organic and functional. IBD consists of Crohn's disease and ulcerative colitis are organic digestive tract diseases characterized by chronic relapse and remittance of intestinal inflammation. The most frequent symptoms of IBD are abdominal pain, diarrhea, gastrointestinal bleeding as well as malnutrition (15). Although there have been several studies regarding to relationship between various gastroenterological disease and urological problems, there has not been a study examining overactive bladder in spectrum of gastroenterological diseases.

In a study by Haim et al. (16) the researchers found that $24.7 \%$ of the patients with Crohn's disease have urological symptoms which included cystitis, hydronephrosis, urolithiasis, enterovesical fistulas and retroperitoneal abscess. In a recent study by Xia et al. (17) authors investigate the mechanism of bladder hypersensitivity in patients with colonic inflammation. In their experimental study with rats, authors found that colon to bladder cross-sensitization exists with the upregulation of brainderived neutrophic factor (BDNF) in dorsal the dorsal root ganglia.

Experimental animal studies have shown that chemically induced $\mathrm{OAB}$ might result in a hypersensitized colon and conversely induced colitis might alter bladder functions (18). There have been several studies evaluating the association between irritable bowel syndrome and overactive bladder $(19,20)$. Matsumoto et al. (19) found that $33.3 \%$ of patients with OAB had concurrent IBS. And they concluded that assessing the defecation habits of patients is important when diagnosing or treating OAB. In contrast with Matsumoto et al., Kim et al. (20) reported no association between IBS and OAB. In our study, OAB was found to be present at $21.6 \%$ of our patient cohort which indicates the increased frequency of OAB compared to healthy population. These results might reflect the aforementioned mechanisms of cross-sensitization between abdominal organs. In concordance with the aforementioned studies, we found a high incidence of $\mathrm{OAB}$ in our patient cohort having gastrointestinal disorders. Although, the relationship between bowel diseases and $\mathrm{OAB}$ have been frequently studied, there is a lack of data assessing the possible relation between hepatic diseases and OAB. Almost all studies, reported a possible relationship based on the similar etiological risk factors for fatty liver and atherosclerosis. 
Our study has limitations. The main limitation of the current study is that the heterogenous nature of the study population like some were in menopause and some were active smoker which might affect bladder habitus. Also, there was no so much patient in gastroenterological disease's subgroups that might cause statistical weakness.

\section{CONCLUSION}

We found a similar overactive bladder frequency in patients having various gastroenterological disease such as hepatitis, inflammatory bowel disease, celiac and autoimmune hepatitis. The incidence of $\mathrm{OAB}$ in our patient cohort was higher than the reported incidence by that evaluating the patients with gastrointestinal disorders in terms of overactive bladder on routine follow-up might be suggested.

\section{ETHICAL DECLARATIONS}

Ethics Committee Approval: The study was carried out with the permission of Health Sciences University Ankara Yüksek İhtisas Training and Research Hospital Ethics Committee (Date: 28.12.2018, Decision No: 65).

Informed Consent: Because the study was designed retrospectively, no written informed consent form was obtained from patients.

Referee Evaluation Process: Externally peer-reviewed.

Conflict of Interest Statement: The authors have no conflicts of interest to declare.

Financial Disclosure: The authors declared that this study has received no financial support.

Author Contributions: All of the authors declare that they have all participated in the design, execution, and analysis of the paper and that they have approved the final version

\section{REFERENCES}

1. Gormley EA, Lightner DJ, Faraday M, and Vasavada SP; Diagnosis and treatment of overactive bladder (non-neurogenic) in adults: AUA/SUFU guideline amendment. J Urol 2015; 193: 1572-80.

2. Jaffe WI and Te AE; Overactive bladder in the male patient: epidemiology, etiology, evaluation, and treatment. Curr Urol Rep 2005; 6: 410-8.

3. Sales-Campos H, Basso PJ, Alves VBF, et al. Classical and recent advances in the treatment of inflammatory bowel diseases. Brazilian J Med Biol Res 2015; 48: 96-107.

4. Karabay EA, Küçükünal NA, Altunay IK, Çerman AA, Alkim C. Gastroenteroloji kliniginde yatan hastalarda izlenen deri bulguları/Skin findings of patients hospitalised in the gastroenterology department. Şişli Etfal Hastanesi Tip Bülteni 2016; 50: 273 .

5. Burisch J, Jess T, Martinato M, and Lakatos PL. The burden of inflammatory bowel disease in Europe. J Crohn's and Colitis 2013; 7: 322-37.
6. Uzun H, Oğullar S, Ünal H, Zorba OÜ, Yazar S, Kalkan M. Nonalcoholic fatty liver disease is associated with benign prostate hyperplasia in men and with overactive bladder in women. Scandinavian J Urol 2013; 47: 497-502.

7. Cetin EG, Demir N, and Sen I. The relationship between insulin resistance and liver damage in non-alcoholic fatty liver patients. Med Bull Sisli Etfal Hospital 2020; 54: 411.

8. Tarcan T, Mangir N, O’zgur M, Akbal C. OAB-V8 Aşırı aktif mesane sorgulama formu validasyon çalışması. Üroloji Bülteni 2012; 21: 113-6.

9. Coyne KS, Zyczynski T, Margolis MK, Elinoff V, Roberts RG. Validation of an overactive bladder awareness tool for use in primary care settings. Adv Ther 2005; 22: 381-94.

10. Tomaszewski J. Postmenopausal overactive bladder. Przeglad Menopauzalny= Menopause Rev 2014; 13: 313 .

11. Kawahara T, Ito H, Yao M, Uemura H. Impact of smoking habit on overactive bladder symptoms and incontinence in women. Int J Urol 2020; 27: 1078-86.

12. Sarikaya K, Senocak C, Sadioglu FE, Ciftci M, Bozkurt OF. Comparison of long-term outcomes of transobturator rectus fascia sling and polypropylene mesh in obese women? J Gynecol Surg 2021; 37: 246-51.

13. Brumovsky PR and Gebhart GF. Visceral organ cross-sensitization - an integrated perspective. Autonomic Neurosci 2010; 153: 10615 .

14. Whorwell PJ, McCallum M, Creed FH, and Roberts CT. Noncolonic features of irritable bowel syndrome. Gut 1986; 27: 37-40.

15. Grundy L, Brierley SM. Cross-organ sensitization between the colon and bladder: to pee or not to pee? Am J Physiol Gastrointest Liver Physiol 2018; 314: 301-8.

16. Ben-Ami H, Ginesin Y, Behar DM, Fisher D, Edoute Y, Lavy A. Diagnosis and treatment of urinary tract complication in Crohn's disease: an experience over 15 years. Can J Gastroenterol 2002; 16: 225-9.

17.Xia C, Shen S, Hashmi F, and Qiao L-Y. Colitis-induced bladder afferent neuronal activation is regulated by BDNF through PLC $\gamma$ pathway. Exper Neurol 2016; 285: 126-35.

18. Pezzone MA, Liang R, and Fraser MO. A model of neural crosstalk and irritation in the pelvis: implications for the overlap of chronic pelvic pain disorders. Gastroenterol 2005; 128: 1953-64.

19. Matsumoto S, Hashizume K, Wada N, et al. Relationship between overactive bladder and irritable bowel syndrome: a large-scale internet survey in Japan using the overactive bladder symptom score and Rome III criteria. BJU Int 2013; 111: 647-52.

20. Kim KS, Kim HJ, Lee SH, Cho ST, and Moon HS. Association between irritable Bowel syndrome and overactive bladder: a research survey. Urology 2017; 109: 88-93. 